\title{
VARIACIONES ESPACIALES Y TEMPORALES DEL ZOOPLANCTON EN UN LAGO MONOMÍCTICO EUTRÓFICO (LAGO TON-TON, URUGUAY)
}

\author{
Daniel Fabián* y Luis Cruz-Pizarro** \\ * Sección Limnología. Facultad de Ciencias. Universidad de la República. Montevideo CP 11400. Uruguay \\ ** Instituto del Agua. Universidad de Granada. 18071 Granada. España
}

Palabras claves: zooplancton, estructura, distribución horizontal, variaciones temporales, sistema monomíctico eutrófico

Key words: zooplankton, structure, spatial distribution, seasonal variation, eutrophic monomictic system.

\section{ABSTRACT \\ SPATIAL AND TEMPORAL VARIATIONS OF ZOOPLANKTON IN AN EUTROPHIC MONOMICTIC LAKE (LAKE TON- TON, URUGUAY).}

The present study was carried out in an eutrophic inonomictic system, lake "Ton-Ton", (Canelones, Uruguay). The main objective was to describe the zooplankton coinmunity structure (specific composition, population densities, horizontal distribution) and seasonal variation as well as the relationships with selected environmental factors Three Copepod species (one Calanoid and two Cyclopoids), seven species of Cladocerans and thirteen genera of Rotifers were found. The zooplanktonic assemblage showed a sharp seasonal cyclc primarily related to mixing and stratification periods. Maximum abundances were registered in spring (and in a lesser extent in autumn) while minimum population densities were observed during summer time.

Copepods and Rotifers were the dominant groups. Notodiaptomus incompositus was the main species, both because of its abundance and presence throughout the year. Dominant Cladocerans species were: Ceriodaphnia dubia, Bosmina huaronensis and Diaphanosoma birgei. No clear differences in either specific composition nor abundance were observed between sampling stations. However, differences in abundance between sampling depths were found. Lake Ton-Ton depicted several characteristics linked to eutrophic systems: great variation in total zooplankton abundance, presence of "key" species, and doininance of small and inedium forms of planktonic herbivores. Physical-chemical variables seemed not to influence directly the zooplanktonic seasonal fluctuations. Other factors such as food quality, predation by vertebrate and invertebrates, as well as intra and interspecific competition may likely explain the spatial and temporal distribution of the zooplanktonic community.

\section{INTRODUCCION}

Al menos, dos razones básicas e íntimamente relacionadas pueden ser sugeridas para justificar el interés por los estudios sobre la estructura y el funcionamiento de la comunidad de zooplancton en sistemas acuáticos continentales:

1. Por motivos teóricos: El zooplancton ocupa una posición pivotal en la cadena trófica pelágica, entre las algas (y las bacterias) y los depredadores de niveles tróficos superiores. La alimentación del zooplancton constituye la principal ruta para el flujo de la energía en ecosistemas limnéticos y representa una fuente de "pérdidas" para los niveles constituidos por el fitoplancton y el bacterioplancton. A la vez, el zooplancton es presa preferida para vertebrados e invetrebrados y constituye un alimento esencial para, por ejemplo, los peces durante las etapas cruciales de reclutamiento.

2. Por razones prácticas: La calidad del agua es el resultado de impactos externos sobre los ecosistemas y de sus transformaciones, a través de interaceiones complejas, en el seno del ambiente acuático. Es por eso por lo que dos estrategias principales se han empleado en la gestión de la calidad de las aguas: a) la reducción de la carga externa de nutrientes y/o la 
aceleración de la salida de nutrientes desde el sistema y b) el control de procesos ecológicos internos (ecotecnologías) de entre las cuales se considera la biomanipulación como la explotación deliberada de las interacciones entre los componentes de los sistemas acuáticos con el fin de reducir la biomasa algal.

Los trabajos pioneros de HRBACEK et al. (1961) y BROOKS \& DODSON (1965) ponen el énfasis en el papel de los peces planctívoros en la estructuración de las comunidades planctónicas y BROOKS \& DODSON (1965) formulan la conocida "Hipotesis de la Eficiencia de Tamaño" (SEH) para explicar la regulación de la estructura de las comunidades de zooplancton (ver también, HALL et ul., 1976) que, en esencia, destaca la importancia de consumo herbívoro por parte del zooplancton y de la depredación de éstos por parte de los peces como las "fuerzas" de la cadena trófica que, en última instancia, regulan la biomasa de fitoplancton y su sucesión estacional.

A pesar de que Uruguay presenta un número considerable de sistemas lénticos naturales y artificiales, los estudios sobre el zooplancton han sido escasos, reduciéndose, casi exclusivamente, a los trabajos de CANZANI \& VARELA (1984) en la laguna Briozzo y a los de FABIÁN \& SCHINCA (1990) y FABIÁN (1993) que estudian la distribución vertical de los Cladóceros en el lago Ton-Ton y la estructura y dinámica de los Crustáceos del plancton del embalse del Cisne, respectivamente.

En el presente trabajo se describe la estructura, distribución horizontal y ciclo estacional de la comunidad zooplanctónica del lago Ton-Ton, un sistema eutrófico cuya primera caracterización físico-química fue realizada por SOMMARUGA (1987) quién además, describe la dinámica de la interface agua-sedimento y en el que DE LEÓN (1993) ha estudiado diferentes aspectos sobre la ccología del fitoplancton.

\section{ÁREA DE ESTUDIO}

El lago Ton-Ton ( $34^{\circ} 51^{\prime}$ S, $56^{\circ} 02^{\prime} \mathrm{W}$ ) (Fig.1) es un sistema reciente (1942) y artificial. No posee afluentes ni efluentes visibles. A pesar de sus dimensiones (Área: 13.6 Há; Zmax: 8 m) se asemeja en su comportamiento a un lago típico y, entre otras, desarrolla una termoclina manifiesta (SOMMARUGA, 1987). De acuerdo con la clasificación propuesta por LEWIS (1983), este sistema se puede caracterizar como monomíctico cálido, presentando estratificación desde finales de la primavera hasta mediados de otoño. Durante el período de estratificación la proximidad del sedimento llega a ser anóxica (SOMMARUGA, 1987, FABIÁN \& SCHINCA, 1990).

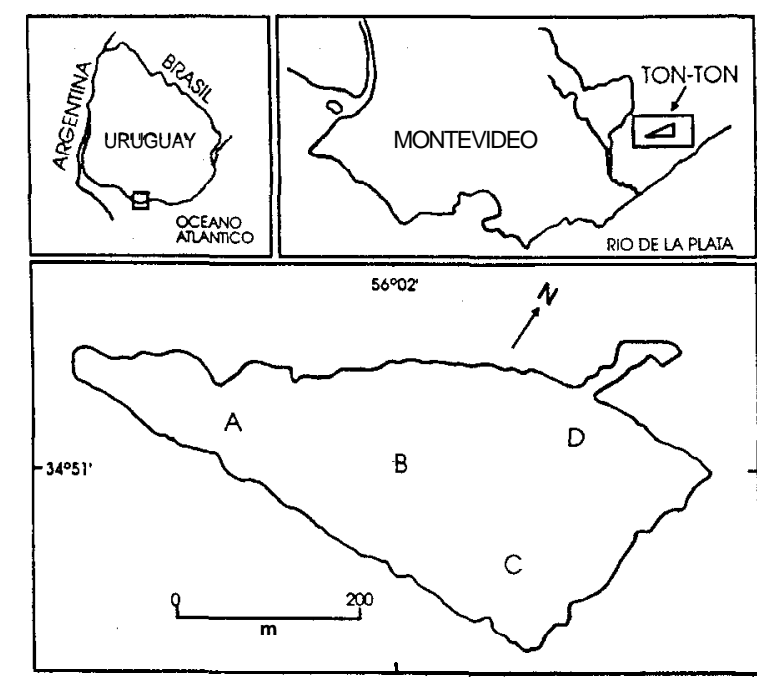

FIGURA 1. Localización del lago Ton-Ton y estaciones de niuestreo. FIGURE 1. Location of Ton-Ton lake and sampling stations.

SOMMARUGA (1987) lo considera como un lago de elevado nivel de eutrofia, sobre la base de características como: una elevada demanda de oxígeno hipolimnético; el desarrollo de floraciones de Anabaenu sp.; la inversión de la relación N:P durante el período de estratificación o la naturaleza del sedimento (fangos de diatomeas, ricos en materia orgánica).

\section{MATERIAL Y MÉTODOS}

El muestreo se realizó con periodicidad mensual, durante el período comprendido entre Julio de 1988 y Junio de 1989, en 4 estaciones situadas en la zona limnética (Fig.1). Las muestras de zooplancton se tomaron a 1 y $4 \mathrm{~m}$ de profundidad, los dos "estratos" más representativos en cuanto a la abundancia de organismos planctónicos, especialmente Cladóceros (FABIÁN \& SCHINCA, 1990). La profundidad de $4 \mathrm{~m}$ se sitúa, además, en el límite superior del metalimnion durante el período de estratificación (SOMMARUGA, 1987; FABIÁN \& SCHINCA, 1990) y efectivamente representa una zona de acumulación de organismos y una barrera a la sedimentación.

Las muestras fueron recolectadas entre el mediodía y las primeras horas de la tarde, utilizando una "trampa" de Schindler de 281 , provista de una malla de $80 \mu \mathrm{m}$. Además de los muestreos cuantitativos, se obtuvieron muestras cualitativas, mediante una red de $120 \mu \mathrm{m}$ de diámetro de malla. Las muestras fueron preservadas "in situ" con formaldehído azucarado (HANEY \& HALL, 1973). El recuento de organismos de las diferentes especies (y estadios de desarrollo, 
en el caso de los Copépodos) se efectuó bajo microscopio binocular convencional a 40 y 100 aumentos en cámaras de tipo Sedgwick-Rafter de $5 \mathrm{~cm}^{3}$ y, los resultados, se expresaron como individuos por metro cúbico. Los microcrustáceos fueron identificados hasta nivel específico y, de género en el caso de los Rotíferos. La diversidad específica fue calculada siguiendo a SHANNON \& WEAVER (1963) y el índice de similitud faunística, entre las estaciones de muestreo, se obtuvo de acuerdo a SORENSEN (1948).

Para cada uno de los días, estaciones y profundidades de muestreo, se midió, in situ las siguientes variables: temperatura y oxígeno disuelto (oxímetro YSI provisto de termistor acoplado), conductividad (conductivímetro Horizon), $\mathrm{pH}$ (pHmetro Digisense) y transparencia (disco de Secchi de $20 \mathrm{~cm}$ de diámetro) y, mediante una botella tomamuestras de Van Dom de 6 litros de capacidad, se obtuvieron muestras de agua para el análisis de las siguientes variables: sólidos en suspensión, materia orgánica en suspensión, ortofosfato reactivo soluble, fósforo total, nitratos y amonio siguiendo una metodología convencional descrita en detalle en APHA (1989) y WETZEL \& LIKENS (1991), entre otros, y clorofila "a" siguiendo el método de Lorenzen, modificado (HOLM-HANSEN \& RIEMAN, 1978).

Los datos sobre velocidad del viento y precipitación fueron suministrados por la Dirección General de Meteorología y corresponden a la estación próxima al cuerpo de agua en estudio.

Para determinar diferencias en abundancia entre las estaciones de muestreo se llevó a cabo un análisis de varianza multidimensional en bloques (SOKAL \& ROHLF, 1981). Se realizó un Análisis de Componentes Principales utilizando la matriz de correlación de los datos transformados logarítmicamente $(\operatorname{Ig} \mathrm{x}+1)$, excepto, obviamente, el $\mathrm{pH}$. El tratamiento de los resultados se realizó de manera conjunta con los datos físico-químicos y biológicos correspondientes a las observaciones de la estación central (B).

\section{RESULTADOS}

Debido a su reducido tamaño, el lago Ton-Ton presenta escasas diferencias entre estaciones de muestreo para las distintas variables medidas, por lo que la Tabla 1 resume los resultados obtenidos como promedio de las profundidades estudiadas, en la estación central (B).

\section{Variables Físicas}

Los vientos predominantes sobre el sistema proceden del sureste, excepto en otoño en que prevalecieron vientos del suroeste. Los valores máximos de velocidad del viento se registraron en Agosto y en Marzo, alcanzándose promedios de $29 \mathrm{Km} / \mathrm{h}$.

La precipitación máxima se registró en primavera y otoño con valores totales estacionales de $269.9 \mathrm{~mm}$ y $243.2 \mathrm{~mm}$, respectivamente (Fig.2). Las oscilaciones del nivel del agua se relacionaron principalmente con los períodos secos y lluviosos, midiéndose una amplitud máxima de $0.72 \mathrm{~m}$, que representa un $10 \%$ de la profundidad máxima del lago.

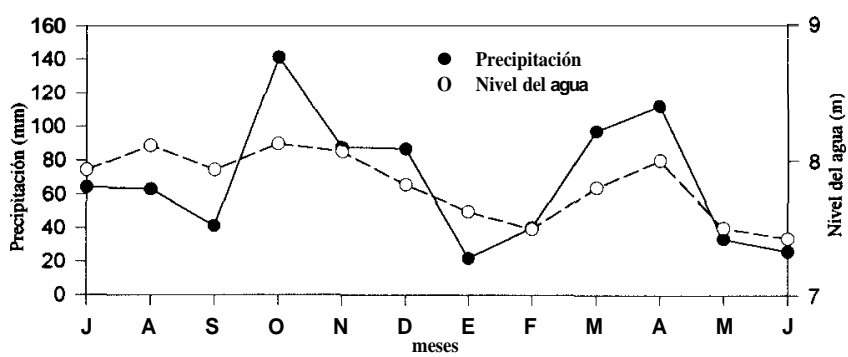

FIGURA 2. Precipitación media mensual y nivel del agua durante el periodo de estudio.

FIGURE 2. Monthly average precipitation and water level during the study period.

Los valores de profundidad de visión del disco de Secchi oscilaron entre $0.96 \mathrm{~m}$ en Diciembre y $2.7 \mathrm{~m}$ en Octubre (Fig.3).

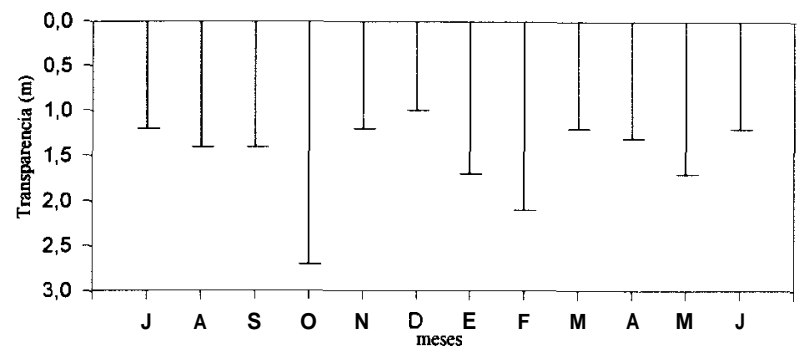

FIGURA 3. Transparencia media mensual del agua durante el periodo de estudio FIGURE 3. Monthly average water transparency dunng the study period.

La temperatura del agua fluctuó entre $10.8^{\circ} \mathrm{C}$ en Agosto y $25.3^{\circ} \mathrm{C}$ en Enero (Tabla 1). En primavera y verano se apreciaron diferencias térmicas entre las profundidades muestreadas, con máximos de $4.4^{\circ} \mathrm{C}$ en Noviembre y mínimos de $1.4^{\circ} \mathrm{C}$ en Febrero. A pesar de carecer de perfiles verticales detallados de temperatura, estas diferencias sugieren una estratificación térmica en el sistema, algo que ya había sido constatado, de forma reiterada, por SOMMARUGA (1987), FABIÁN \& SCHINCA (1990) y DE LEÓN (1993). 
TABLA I. Parámetros fisicoquímicos y biológicos correspondientes a la estación central (B), valores medios, máximos, mínimos y desvío estandar de las observaciones. TABLE I. Physico-chemical and biological parameters corresponding to central station (B), average values, maxima, minima and standard deviation of the observations.

\begin{tabular}{|c|c|c|c|c|c|c|c|c|c|c|}
\hline Meses & $\begin{array}{c}T \\
(\alpha C)\end{array}$ & $\begin{array}{c}02 \\
(\mathrm{mg} / \mathrm{l}) \\
\end{array}$ & $\mathrm{pH}$ & $\begin{array}{c}\text { Cond. } \\
(\mu \mathrm{S} / \mathrm{cm})\end{array}$ & $\begin{array}{c}\text { STS } \\
(\mathrm{mg} / \mathrm{l}) \\
\end{array}$ & $\begin{array}{l}\text { MO } \\
(8) \\
\end{array}$ & $\begin{array}{c}\mathrm{NH4} \\
(\mathrm{mg} / \mathrm{l})\end{array}$ & $\begin{array}{c}\mathrm{N03} \\
(\mathrm{mg} / \mathrm{l}) \\
\end{array}$ & $\begin{array}{c}\mathrm{PO4} \\
(\mathrm{mg} / \mathrm{l}) \\
\end{array}$ & $\begin{array}{c}\mathrm{PT} \\
(\mathrm{mg} / \mathrm{l}) \\
\end{array}$ \\
\hline $\begin{array}{l}\text { Julio } \\
\end{array}$ & 11.0 & 8.2 & 6.8 & 320 & 3.0 & 79 & 3.2 & $11 . T$ & 1.1 & 2.2 \\
\hline Agosto & 10.8 & 10 & 7.1 & 375 & 3.8 & 77 & 2.8 & 23.5 & 0.5 & 1.2 \\
\hline Setiembre & 14.0 & 9.7 & 8.3 & 425 & 3.2 & 53 & 5.9 & 15.2 & 0.7 & 0.9 \\
\hline Octubre & 17.0 & 7.6 & 7.2 & 450 & 1.4 & 68 & 2.1 & 2.9 & 1.2 & 1.8 \\
\hline Noviembre & 22.0 & 7.5 & 7.2 & 450 & 8.6 & 90 & 3.7 & 1.4 & 2.6 & 4.7 \\
\hline Diciembre & 23.0 & 7.0 & 7.8 & 435 & 5.2 & 70 & 8.3 & 4.2 & 3.5 & 7.4 \\
\hline Enero & 25.3 & 7.3 & 7.8 & 510 & 1.3 & 53 & 12.9 & 3.4 & 6.4 & 8.8 \\
\hline Febrero & 24.3 & 6.1 & 7.4 & 405 & 3.9 & 57 & 13.6 & 2.7 & 4.2 & 10.2 \\
\hline Marzo & 22.0 & 6.8 & 8.0 & 305 & 2.7 & 68 & 13.0 & 4.2 & 3.3 & 12.1 \\
\hline Abril & 19.5 & 6.1 & 7.5 & 340 & 2.7 & 57 & 4.3 & 5.6 & 1.6 & 2.6 \\
\hline Mayo & 15.0 & 7.0 & 7.1 & 400 & 3.4 & 23 & 3.5 & 6.5 & 1.1 & 2.2 \\
\hline Junio & 13.3 & 8.8 & 7.4 & 500 & 2.0 & 80 & 3.3 & 7.4 & 1.8 & 3.4 \\
\hline Máximo & 25.3 & 10.0 & 8.3 & 510.0 & 8.6 & 90.0 & 13.6 & 23.5 & 6.4 & 12.1 \\
\hline Mínimo & 10.8 & 6.1 & 6.8 & 305.0. & 1.3 & 23.0 & 2.1 & 1.4 & 0.5 & 0.9 \\
\hline Desv.Est & 5.2 & 1.3 & 0.4 & 65.7 & 2.0 & 17.6 & 4.4 & 6.4 & 1.7 & 3.8 \\
\hline
\end{tabular}

\section{Variables Químicas}

La concentración media de oxígeno disuelto en el perfil muestreado, osciló entre 6.1 y 10.0 mgll. (Tabla 1). Tan sólo se observaron diferencias significativas entre las profundidades de muestreo durante los meses de verano, una época en la que tiene lugar una marcada reducción de la concentración de oxígeno y se llegan a medir tensiones de $2.0 \mathrm{mg} / \mathrm{l}$ a $4 \mathrm{~m}$. Sobre una base anual, los valores promedio de concentración de oxígeno disuelto (y, evidentemente, del porcentaje de saturación de oxígeno) mostraron correlación negativa con los de temperatura $(r=-0.825$ y $r=-0.892 ; p<0.01$ para los períodos de estratificación y mezcla, respectivamente).

El pH se mantuvo ligeramente alcalino a lo largo del año, con valores promedio de entre 6.8 y 8.3 (Tabla 1 ).

La conductividad eléctrica varió entre 305 y $510 \mu{\mathrm{S} . \mathrm{cm}^{-1}}^{-1}$ (Tabla 1).

Los nitratos, que mostraron concentraciones promedio en la columna de agua muestreada de entre 1.4 y $23.5 \mathrm{mg} / \mathrm{l}$, representaron la forma predominante de nitrógeno durante el período de mezcla mientras que durante los meses de verano, el amonio (con valores promedio de entre 8.3 y $13.6 \mathrm{mgll}$ ) fue la forma dominante.

Las concentraciones de fósforo total y de ortofosfato reactivo soluble alcanzaron, igual que en el caso del amonio, máximos valores durante el período de estratificación. Las concentraciones medias en el perfil vertical muestreado oscilaron durante el período de estudio entre 0.9 y $12.1 \mathrm{mg} / 1 \mathrm{y}$ entre 0.5 y $6.4 \mathrm{mg} / \mathrm{l}$, respectivamente.

\section{Variables Biológicas}

La concentración de sólidos en suspensión fluctuó entre 1.3 $\mathrm{mg} / \mathrm{l}$ en Enero y $8.6 \mathrm{mg} / \mathrm{l}$ en Noviembre, cuando se registraron los valores más elevados de biomasa algal (Tabla 1). De hecho, la evolución anual de los valores promedio de estas dos variables (sólidos en suspensión y clorofila) mostró una correlación positiva significativa $(r=0.616 ; p<0.05)$. El porcentaje (en peso) de materia orgánica superó el 50\% del de la materia en suspensión, prácticamente durante todo el período de estudio.

La concentración de clorofila presentó una clara estacionalidad con valores más elevados durante el período de estratificación (Fig.4). Los valores máximos, medidos a la profundidad de $1 \mathrm{~m}$, se registraron durante los meses de Noviembre (50.2 mg.m ${ }^{-3}$ ) y Diciembre $\left(51.8 \mathrm{mg} \cdot \mathrm{m}^{-3}\right)$. A partir de entonces se observa una tendencia decreciente en la concentración hasta alcanzar el valor mínimo en el muestreo correspondiente al mes de Abril (3.0 mg.m $\left.\mathrm{m}^{-3}\right)$. En Mayo, coincidiendo con el comienzo del período de circulación, la 
concentración se incrementó en un orden de magnitud (31.2 mg. $\mathrm{m}^{-3}$ ). Los valores medidos a $4 \mathrm{~m}$ de profundidad fueron notoriamente inferiores'a los registrados en superficie, aunque su evolución anual fue similar.

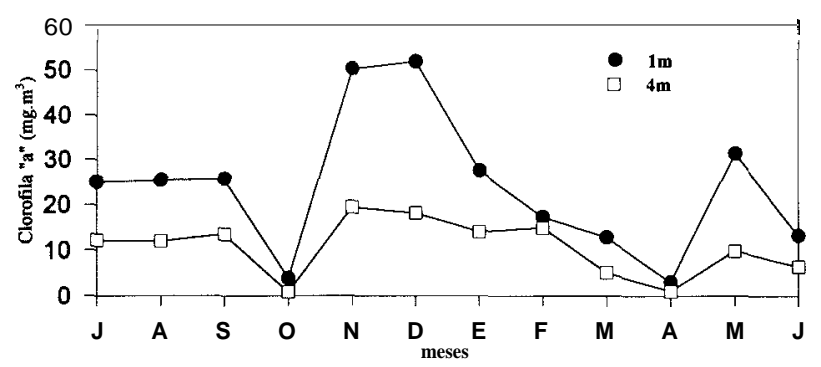

FIGURA 4. Variación espacio-temporalde la clorofila "a" durante el período de estudio (estación B).

FIGURE 4. Spatial and temporal variation of chlorophyll "a" during the study period.

\section{Comunidad zooplanctónica}

La comunidad zooplanctónica está representada por 3 especies de Copépodos (2 Ciclopoides y 1 Calanoide), 7 especies de Cladóceros y 13 géneros de Rotíferos que, salvo para las estimas de diversidad y el análisis de afinidad faunística, se contabilizaron como un único grupo (Tabla 2).

TABLA II. Composición del zooplancton del lago Ton-Ton. TABLE II. Composition of zooplankton of Ton-Ton lake.

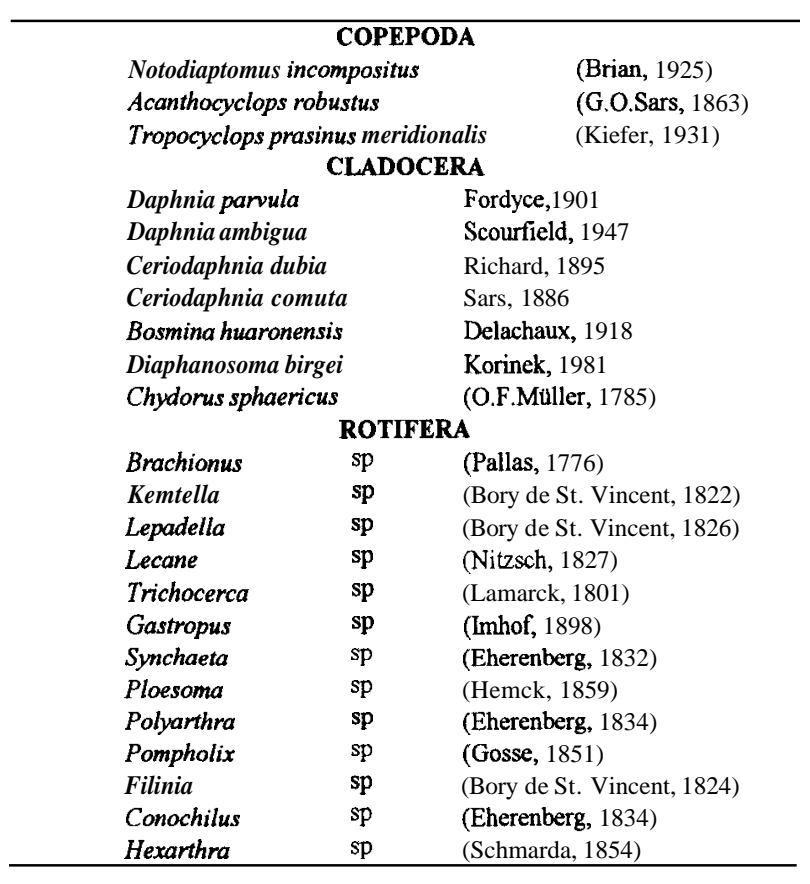

En términos de número de individuos, los Copépodos representaron el $49 \%$ de la comunidad zooplanctónica; los Rotíferos el 38\% y los Cladóceros el 13\%. Como conjunto, la comunidad zooplanctónica mostró un evidente patrón estacional, con máximas abundancias poblacionales en primavera y mínimas en verano (Fig.5). Este patrón se repite en cada uno de los grupos, a excepción de los Ciclopoides, que mostraron máximos invernales y de los Cladóceros (especialmente Diaphanosoma birgei) que exhiben, además, un segundo máximo estival. La aplicación del test de Wilks a los datos sobre abundancias poblacionales, mostró diferencias significativas entre profundidades $(\mathrm{F}$ aprox. $=\mathrm{F}(10,68)=4.6 ; \mathrm{p}$ $=0.0001)$, pero no entre estaciones de muestreo $(\mathrm{F}$ aprox. $=$ $\mathrm{F}(30,200)=1.4 ; \mathrm{p}=0.07)$

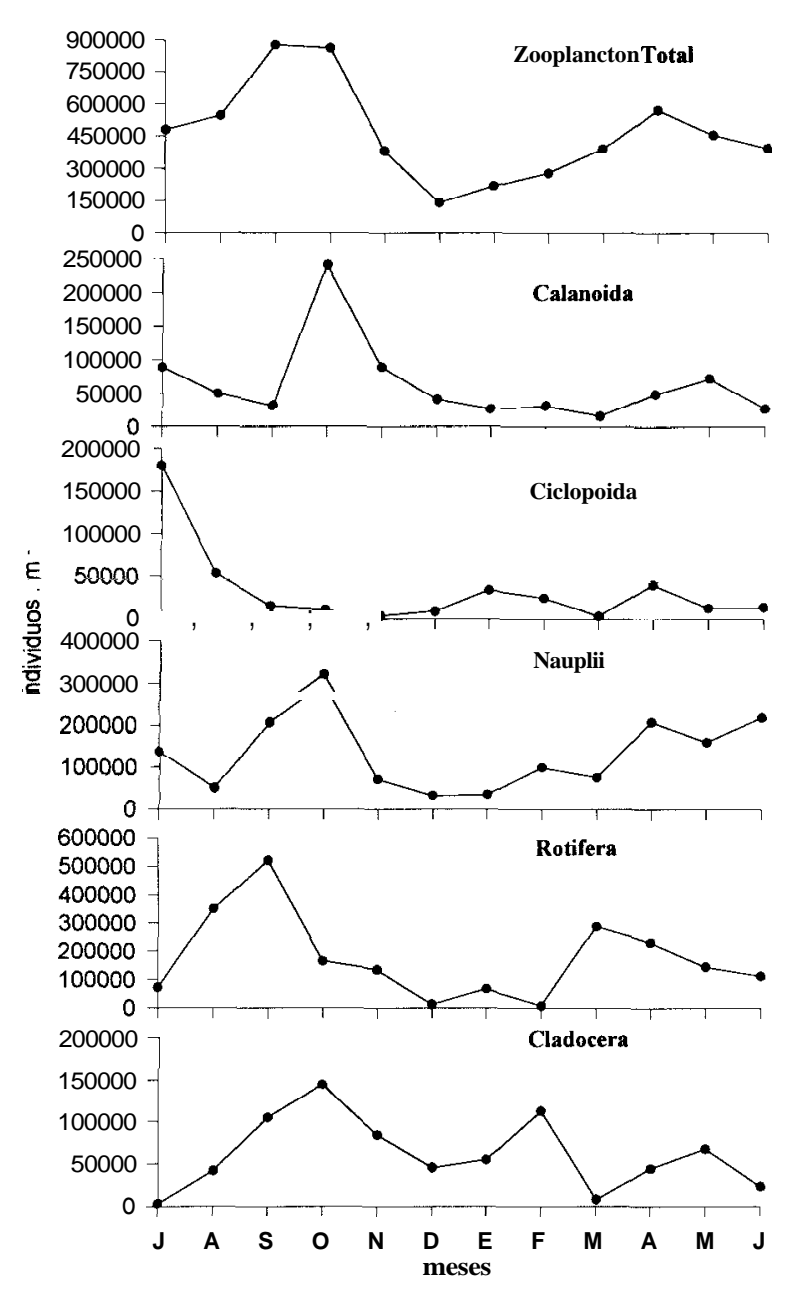

FIGURA 5. Variaciones estacionales de la abundancia media del zooplancton total y los diferentes taxones durante el período de estudio.

FIGURE 5. Seasonal variations of the average abundance of total zooplankton and the differem taxas buing the stady period. 
Notodiaptomus incompositus, la única especie de Calanoide presente, dominó en términos de abundancia durante la mayor parte del período de estudio, siendo, por lo tanto, "responsable" del patrón de variación temporal observado para los Copépodos en conjunto.(Fig.6). La Figura 7 muestra las variaciones en la densidad poblacional de esta especie durante el ciclo anual en las diferentes estaciones y profundidades muestreadas. Como se puede apreciar, los valores máximos de abundancia se registraron a mediados de primavera y, los mínimos, a principios de verano. Es de destacar que durante el período estudiado, los estadios copepodíticos siempre constituyeron la fracción dominante.

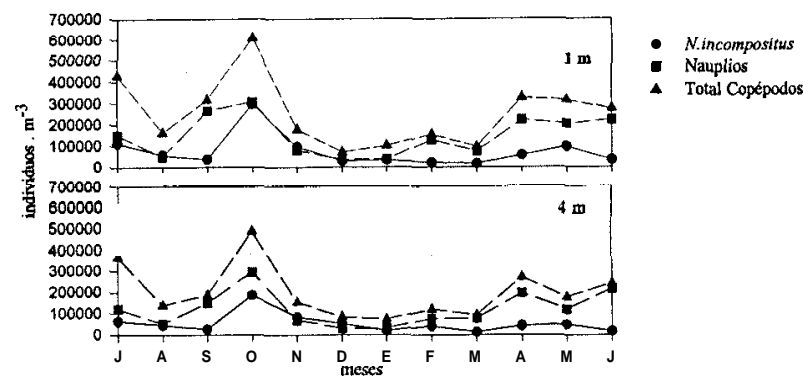

FIGURA 6. Variación de la abundancia media de copépodos, nauplios y $\mathrm{N}$. Incompositus durante el período de estudio.

FIGURE 6. Average abundance variation of copepods. nauplii and $\mathrm{N}$. Incompositus during the study period.

Acanthocyclops robustus y Tropocyclops prasinus rneridionalis son las dos especies de Ciclopoides en el lago. De ellas, A. robustus se observó durante todo el ciclo anual de estudio, con máximos poblacionales en invierno y, mínimos, durante los meses de Febrero y Marzo así como en primavera (Fig.7). Como en el caso de N. incompositus, los estadíos copepodíticos dominaron en la estructura de edad de la población, dando cuenta, por lo tanto, de las variaciones estacionales observadas en la abundancia poblacional.

T. prasinus meridionalis presentó tamaños poblacionales considerablemente inferiores que A. robustus durante buena parte del período en que se encontró en el lago y únicamente cuando se registraron sus máximos de abundancia (Febrero) ambas especies mostraron densidades comparables. A diferencia de las demás especies de Copépodos, las máximas abundancias de $T$. p. rneridionalis corresponden a períodos en que predominaron hembras adultas (Fig.7). Desaparece en otoño.

Ceriodaphnia dubiu, Bosrnina huaronensis y Diaphanosoma birgei, fueron las especies dominantes de



FIGURA 7. Variación espacio-teniporal de las especies de copépodos durante el período de estudio.

FIGURE 7. Spatial and teniporal variation of the copepods species during the study period.

Cladóceros. De ellas, Ceriodaphnia dubia y Bosminu huaronensis, que presentaron abundancias alternadas en el tiempo (Fig.8), fueron las especies mejor representadas, con máximos poblacionales registrados durante la primavera, (especialmente $C$. dubia), si bien B. huaronensis presentó, también, elevadas densidades en verano y otoño (Fig. 9).

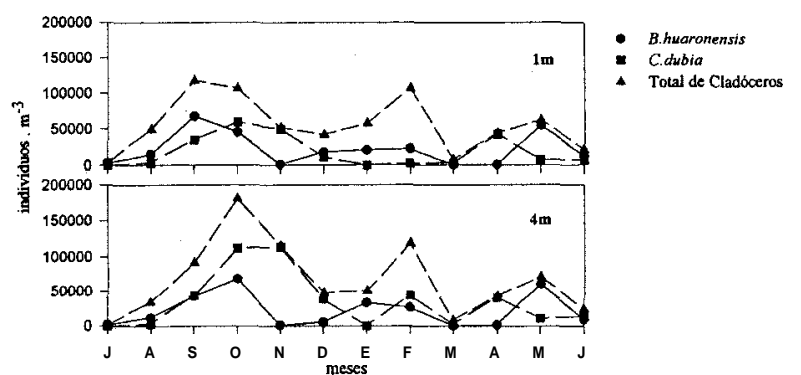

FIGURA 8. Variación de la abundancia niedia de C. Dubia y B. huaronensis durante el período de estudio.

FIGURE 8. Average abundance variation of C. Dubia and B. huaronensi during the study period. 
Daphnia parvula y D. ambigua presentaron sus máximas abundancias poblacionales en invierno (Fig.9) y Ceriodaphnia cornuta mostró una presencia esporádica, encontrándose únicamente en Febrero y Marzo, preferentemente a la mayor profundidad muestreada.

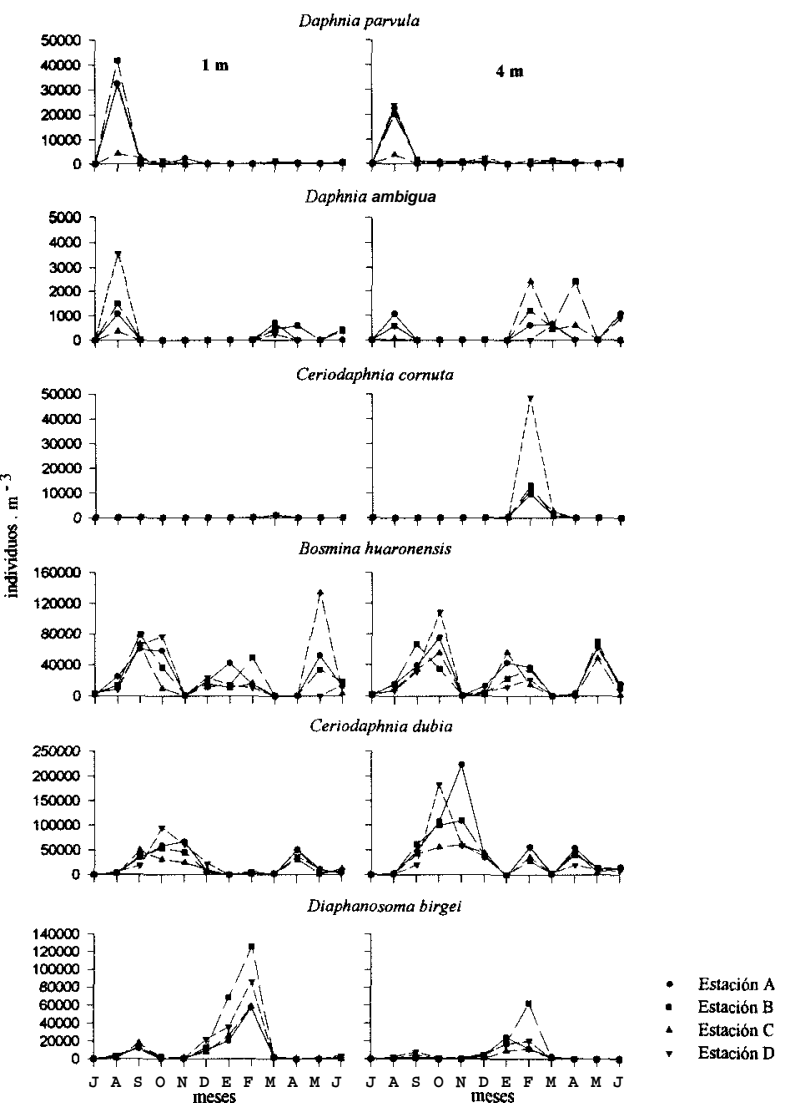

FIGURA 9. Variación espacio-temporal de las especies de cladóceros durante el periodo de estudio.

FIGURE 9. Spatial and temporal variation of the cladocerans species dunng the study period.

Como muestra la Tabla 3 , los valores calculados de diversidad específica fluctuaron entre 0.53 y 2.62 bits/ind.,dentro del rango de los habituales citados en la literatura (PAGGI, 1980). Puntualmente, el valor máximo correspondió a la estación D durante el mes de Febrero, a 4 m y el valor mínimo se obtuvo en esa misma estación y profundidad, durante el mes de Julio. En términos generales, las variaciones de los valores del índice de diversidad entre estaciones fueron comparables a las observadas entre profundidades de muestreo.

El índice de similitud faunística calculado entre estaciones de muestreo fue muy elevado, fluctuando entre un $85 \%$ y un $100 \%$ a $1 \mathrm{~m}$ y entre un $83 \%$ y un $100 \%$ a 4 m (Tabla 4 ).
TABLA IIl. índice de diversidad en base al número de individuos (H) expresado en bits de las diferentesestaciones de muestreo.

TABLE III. Numeric diversity index $(H)$ based on number of individual expressed in bits for the different sampling.

\begin{tabular}{lccccccccccc}
\hline $\mathbf{1} \mathrm{m}$ & A & B & C & D & Media & 4 m & A & B & C & D & Media \\
\hline J几L1O & 1.21 & 1.11 & 118 & 1.10 & 115 & & 1.28 & 1.03 & 1.37 & 053 & 105 \\
AGOSTO & 2.26 & 2.04 & 1.82 & 2.14 & 2.07 & & 2.33 & 2.17 & 2.05 & 2.00 & 2.14 \\
SETIEMBRE & 2.14 & 2.11 & 2.12 & 1.99 & 2.09 & & 1.99 & 2.07 & 2.07 & 2.20 & 2.08 \\
OCTUBRE & 1.51 & 1.25 & 1.02 & 1.21 & 1.25 & & 1.87 & 1.60 & 1.68 & 1.51 & 1.67 \\
NOVEMBRE & 1.25 & 1.18 & 1.07 & 1.14 & 1.16 & & 1.12 & 1.16 & 1.25 & 1.30 & 1.21 \\
DICIEMBRE & 1.87 & 2.08 & 2.45 & 2.36 & 2.19 & & 1.97 & 1.81 & 1.59 & 1.84 & 1.80 \\
ENERO & 2.05 & 1.87 & 2.03 & 1.99 & 1.98 & & 2.22 & 2.37 & 1.96 & 2.30 & 2.21 \\
FEBRERO & 1.87 & 1.68 & 1.84 & 1.87 & 1.82 & & 2.50 & 2.54 & 2.56 & 2.62 & 2.55 \\
MARZO & 1.79 & 1.81 & 1.86 & 1.95 & 1.85 & & 2.06 & 1.96 & 2.09 & 2.28 & 2.10 \\
ABRIL & 1.63 & 1.68 & 1.58 & 1.62 & 1.63 & & 1.69 & 1.83 & 1.59 & 1.69 & 1.70 \\
MAYO & 1.70 & 1.43 & 1.43 & 1.47 & 1.51 & & 1.64 & 1.58 & 1.58 & 1.67 & 1.62 \\
JUNIO & 1.70 & 1.86 & 1.87 & 2.00 & 1.86 & & 1.98 & 1.94 & 1.67 & 2.12 & 1.93 \\
\hline
\end{tabular}

TABLA IV. índice de similitud faunística entre las estaciones de muestreo (valores medios (\%) de las combinaciones entre las estaciones).

TABLE IV. Faunistic similanty index between sampling stations (average values (\%) for the whole possible combinations).

\begin{tabular}{lccc}
\hline Meses & $\mathbf{l m}$ & $\mathbf{4 m}$ & Media \\
\hline JULIO & $\mathbf{9 3}$ & $\mathbf{9 2}$ & $\mathbf{9 3}$ \\
AGOSTO & 100 & 100 & 100 \\
SETIEMBRE & $\mathbf{9 6}$ & $\mathbf{9 2}$ & 94 \\
OCTUBRE & $\mathbf{8 7}$ & $\mathbf{9 1}$ & $\mathbf{8 9}$ \\
NOVIEMBRE & $\mathbf{9 0}$ & $\mathbf{8 3}$ & $\mathbf{8 7}$ \\
DICIEMBRE & 95 & $\mathbf{9 6}$ & 96 \\
ENERO & 100 & $\mathbf{9 0}$ & 95 \\
FEBRERO & 100 & 94 & 97 \\
MARZO & 97 & 97 & 97 \\
ABRIL & $\mathbf{7 6}$ & $\mathbf{7 9}$ & $\mathbf{7 8}$ \\
MAYO & $\mathbf{8 5}$ & 94 & 90 \\
JUNIO & $\mathbf{8 5}$ & $\mathbf{8 3}$ & $\mathbf{8 4}$ \\
\hline
\end{tabular}

Del resultado del análisis de componentes principales llevado a cabo, se desprende que los tres primeros ejes explican en conjunto un $54 \%$ de la varianza total. El primer eje explica el $24 \%$ de la varianza, lo que significa que casi un tercio de la variabilidad total de la matriz de datos puede ser explicada por un solo factor. Como se observa en la Tabla 5 y en la Fig. 10, este primer eje agrupa en su extremo positivo a la temperatura, la concentración de fósforo total y la concentración de ortofosfato reactivo soluble, mientras que la concentración de oxígeno disuelto y de nitratos presentan elevadas correlaciones negativas. 

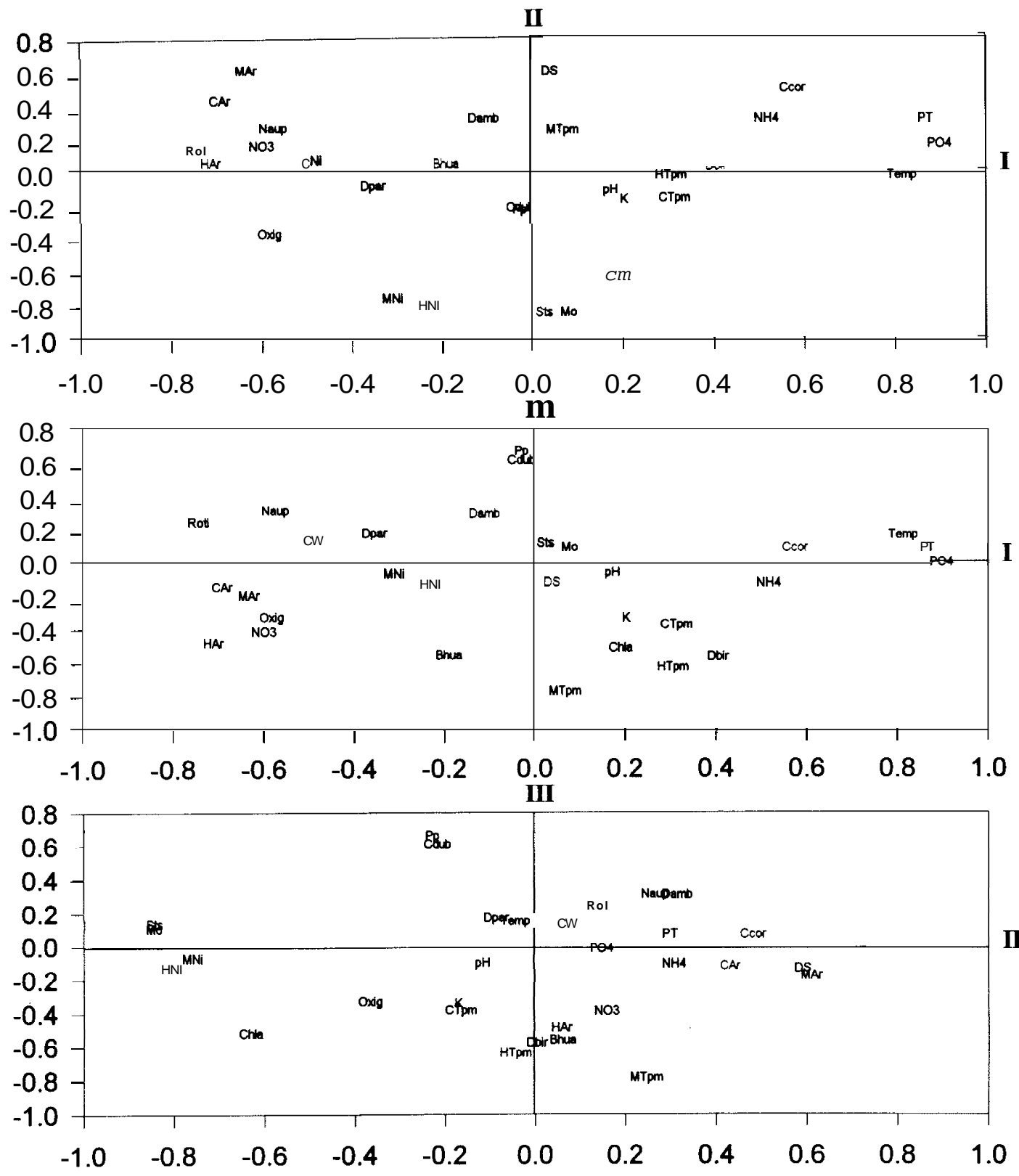

Referencias:

$\begin{array}{ll}\text { HNi:Hembra de N.incompositus } & \text { MTpm:Machos de T.p.meridionalis } \\ \text { MNi:Machos de N.incompositus } & \text { CTpm:Copepoditos de T.p.meridionalis } \\ \text { CNi:Copepoditos de N.incompositus } & \text { Naup:Nauplios } \\ \text { HAr:Hembras de A.robustus } & \text { Roti:Rotiferos } \\ \text { MAr:Machos de A.robustus } & \text { Bhua:B.huaronensis } \\ \text { CAr:Copepoditos de A.robustus } & \text { Dbir:D.birgei } \\ \text { HTpm:Hembras de T.p.meridionalis } & \text { Doar:D.parnula }\end{array}$

Damb:D.ambigua Sts:Ś́lidos totales en suspensión Chla:Clorofila"a" Cdub:C.dubia Mo:Materia orgánica DS:D.Secchi Ccor:C.cornuta Pp:Precipitación

Temp:Temperatura NH4:Amonio

Oxig:Oxigeno NO3:Nitrato

pH PO4:Ortolosfato

HTpm:Hembras de T.p.meridionalis Dear:D.parvula

K:Conductividad PT:Fósforo total

FIGURA 10. Análisis de componentes principales de las variables físico-químicas y biológicas durante el período de estudio. FIGURE 10. Principal component analysis of the physico-chemical and biological variables during the study period. 
TABLA V. Porcentaje de la varianza total explicada por los componentes principales y correlaciones entre ejes y variables.

TABLE V. Percentage of total vanance explained by the principal component and correlations between axes and variables.

\begin{tabular}{|c|c|c|c|c|}
\hline$\overline{\text { Símbolos }}$ & & $\begin{array}{l}\text { Eje I } \\
24 \%\end{array}$ & $\begin{array}{l}\text { Eje II } \\
16 \%\end{array}$ & $\begin{array}{c}\text { Eje III } \\
14 \%\end{array}$ \\
\hline$\overline{\mathbf{H N i}}$ & (Hembras de N. Incompositus) & -0.231 & -0.805 & -0.134 \\
\hline MNi & (Machos de N.incompositus) & -0.312 & -0.759 & -0.072 \\
\hline $\mathbf{C N i}$ & (Copepoditos de N. incompositus) & -0.489 & 0.073 & 0.132 \\
\hline $\mathbf{H A \mathbf { r }}$ & (Hembras de A. robustus) & -0.712 & 0.059 & -0.433 \\
\hline MAr & (Machos de A. robustus) & -0.632 & 0.610 & -0.200 \\
\hline CAr & (Copepoditos de A. robustus) & -0.692 & 0.432 & -0.146 \\
\hline HTpm & (Hembras de T.p.meridionalis) & 0.306 & -0.044 & -0.634 \\
\hline MTpm & (Machos de T.p.meridionalis) & 0.067 & 0.246 & -0.777 \\
\hline CTpm & (Copepoditos de T.p.meridionalis) & 0.314 & -0.165 & -0.379 \\
\hline Naup & (Nauplios) & -0.573 & 0.266 & 0.313 \\
\hline Roti & (Rotíferos) & -0.743 & 0.139 & 0.242 \\
\hline Bhua & (B. Huaronensis) & -0.190 & 0.060 & -0.560 \\
\hline Dbir & (D. birgei) & 0.406 & 0.005 & -0.573 \\
\hline Dpar & (D. parvula) & -0.354 & -0.085 & 0.171 \\
\hline Damb & (D. Ambigua) & -0.108 & 0.315 & 0.309 \\
\hline Cdub & (C.dubia) & -0.027 & -0.216 & 0.609 \\
\hline Ccor & (C.cornuta) & 0.576 & 0.483 & 0.077 \\
\hline Temp & (Temperatura) & 0.815 & -0.043 & 0.154 \\
\hline Oxig & (Oxígeno) & -0.582 & -0.366 & -0.329 \\
\hline $\mathbf{p H}$ & & 0.174 & -0.116 & -0.101 \\
\hline $\mathrm{K}$ & (Conductividad) & 0.203 & -0.171 & -0.341 \\
\hline Sts & (Sólidos totales en suspensión) & 0.026 & -0.844 & 0.132 \\
\hline Mo & (Materia orgánica) & 0.079 & -0.844 & 0.104 \\
\hline $\mathbf{P p}$ & (Precipitaciones) & -0.028 & -0.228 & 0.659 \\
\hline NH4 & (Amonio) & 0.518 & 0.307 & -0.133 \\
\hline N03 & (Nitrato) & -0.600 & 0.159 & -0.413 \\
\hline PO4 & (Ortofosfato) & 0.898 & 0.147 & -0.011 \\
\hline PT & (Fósforo total) & 0.867 & 0.298 & 0.074 \\
\hline Chla & Clorofila"a" & 0.191 & -0.630 & -0.519 \\
\hline DS & (D.Secchi) & 0.039 & 0.591 & -0.159 \\
\hline
\end{tabular}

El segundo eje explica el $16 \%$ de la varianza, esto es, hay una reducción importante de la información asociada a este eje. En su parte positiva se sitúa la visibilidad y, en la parte negativa, la concentración de sólidos en suspensión, la materia orgánica y la concentración de clorofila. El tercer eje, que sólo explica un $14 \%$ de la varianza, muestra correlaciones menores con la mayoría de las variables físico-químicas. La precipitación fue la variable mejor correlacionada con este eje.

En relación con las variables que representan cada una de las especies (y, en su caso, estadios de desarrollo o sexos diferentes) de zooplancton del sistema, el análisis muestra que con el primer eje se correlacionaron positivamente (pero no significativamente) la abundancia de adultos y de estadios copepodíticos de Tropocyclops prasinus rneridionalis, mientras que D. birgei y C. cornuta presentaron una elevada correlación y el resto de las especies, en mayor o menor grado, se correlacionaron negativamente (Tabla 5 y Fig.10). Con el segundo eje, se observa que la mayoría de las especies presentó una baja correlación positiva, excepto las densidades de hembras y de machos de N. incornpositus, con un gran "peso" en su parte negativa (Tabla 5 y Fig.10). Con el tercer eje, la especie que mostró una mayor correlación positiva fue C. dubia. La abundancia de hembras y de machos de T. $p$. meridionalis presentaron altas correlaciones negativas, al igual que B. huaronensis y D. birgei, aunque éstas en menor grado (Tabla 5 y Fig.10).

Finalmente, de la distribución en el plano definido por cada par de ejes de las variables que representan las fechas de 

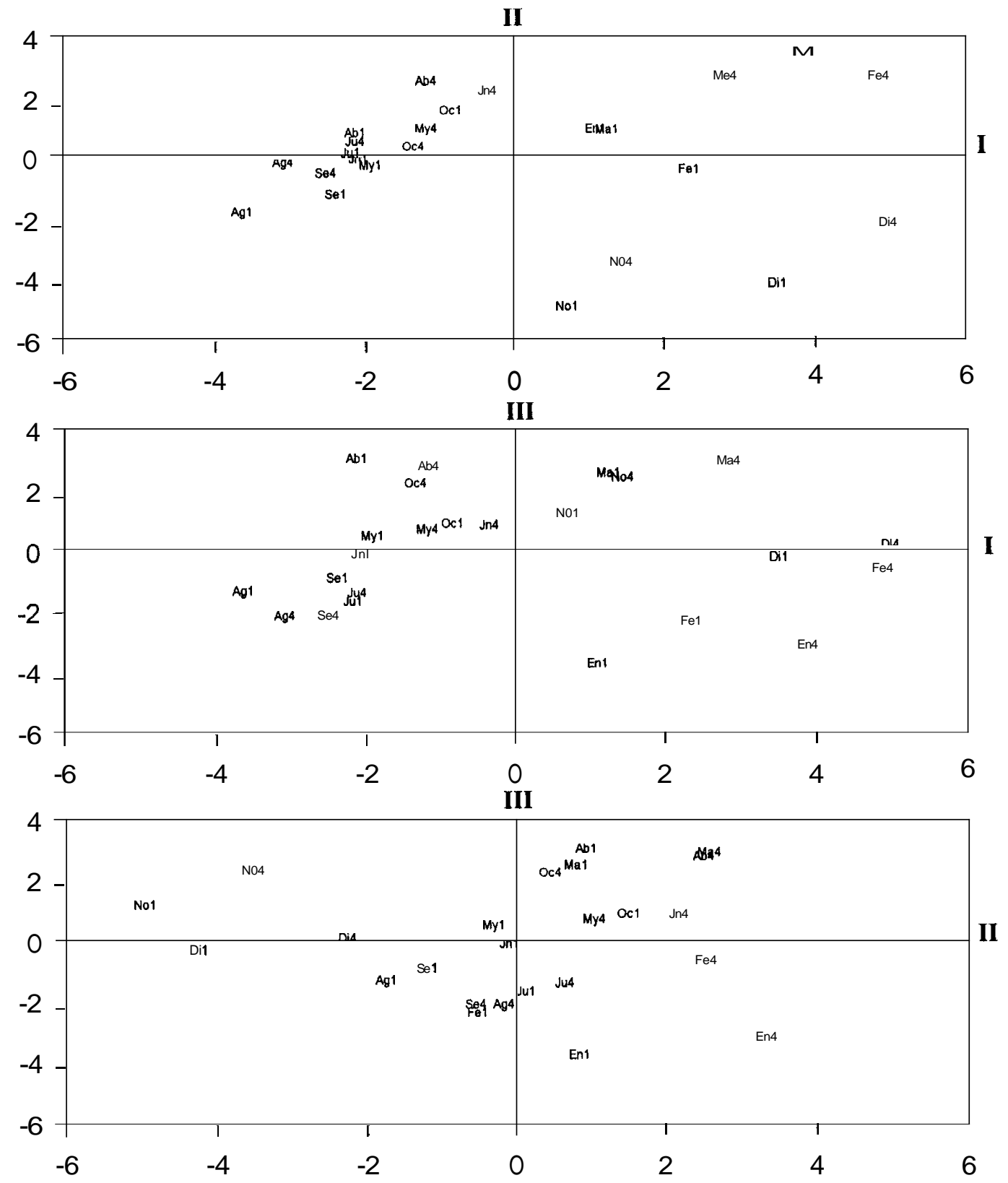

Referencias:

$\begin{array}{lll}\text { Ju1:Julio a I m } & \text { En1:Enero a I m } & \text { Ju4:Julio a 4m } \\ \text { Agl } \mid \text { Agosto a I m } & \text { Fe1:Febrero a Im } & \text { Ag4:Agosto a 4m } \\ \text { Se1:Setiembre a I m } & \text { Ma1:Marzo a Im } & \text { Se4:Sotiembre a 4m } \\ \text { Oc1:Octubre a Im } & \text { Abl Abril a Im } & \text { Oc4:Octubre a 4m } \\ \text { No1:Noviembre a I m } & \text { Ma1:Mayo a I m } & \text { No4:Noviembre a 4m } \\ \text { Di1:Diciembro a I m } & \text { Ju1:Junio a Im } & \text { Di4:Diciembre a 4m }\end{array}$

En4:Enero a $4 \mathrm{~m}$ Fe4:Febrero a $4 \mathrm{~m}$ Ma4:Marzo a $4 \mathrm{~m}$ Ab4:Abril a 4m

Ma4:Mayo a 4m

Ju4:Jurio a $4 m$

FIGURA 11. Análisis de componentes principales de las observaciones durante el período de estudio FIGURE 11. Principal component analysis of the observations during the study period. 
muestreo (los meses, en nuestro caso), destacamos la elevada correlación positiva que presentan las observaciones de Diciembre, Febrero y Enero con el primer eje y, en menor grado, las de Marzo y Noviembre. El resto de las observaciones se sitúan en el extremo negativo de este eje (Fig.11). En este caso resulta evidente la separación de dos grupos sobre la base de la temperatura que se corresponden, a su vez, con períodos de estratificación y mezcla en el sistema a lo largo del año. Por otra parte se observó que en los meses de estratificación térmica se separaron notoriamente las observaciones correspondientes a las dos profundidades muestreadas, evidenciando la heterogeneidad de la columna en este período (Fig.11). Las observaciones que mostraron una mayor correlación negativa con el eje 2 fueron las correspondientes a los meses de Noviembre y Diciembre a la profundidad de $1 \mathrm{~m}$, coincidiendo, como se expone más arriba, con los valores más altos de materia orgánica, sólidos en suspensión y clorofila. En el extremo postivo del eje 3, aparecen agrupadas las observaciones correspondientes a los meses de Marzo, Abril, Octubre y Noviembre, mientras que las de Enero aparecen claramente segregadas en su extremo negativo.

\section{DISCUSIÓN}

Es bien conocido que en los ambientes acuáticos continentales, posiblemente por su carácter más efímero y fluctuante, el plancton animal resulta incomparablemente más pobre y simplificado que el zooplancton marino (MARGALEF, 1983). Por ejemplo, en el trabajo clásico de PENNAK (1957), que recopila información sobre la composición del zooplancton en 42 lagos de pequeño y mediano tamaño en todo el mundo, una «estructura característica» estaría compuesta por 1 a 3 especies de Copépodos, de 2 a 4 especies de Cladóceros y entre 3 y 7 especies de Rotíferos. En comparación con estos resultados promedio, el lago Ton-Ton muestra una elevada riqueza específica y características que, en esencia, responden a lo esperable en un sistema eutrófico: es el caso de su composición específica y estructura de tamaños o de las (notables) fluctuaciones en el número total de individuos.

La dominancia numérica de los Rotíferos en ambientes eutróficos ha sido ampliamente documentada (GANNON \& STEMBERGER, 1978; BLANCHER, 1984; ORCUTT \& PACE, 1984) aunque es cierto que su dominancia en sistemas oligo y mesotróficos (MATSUMURA-TUNDISI \& TUNDISI,1976; MATSUMURA-TUNDISI et al., 1989) parece sugerir que factores como la naturaleza (y el origen) de los lagos y/o aspectos relacionados con la competencia por el alimento, pueden jugar un papel decisivo (MATSUMURA-TUNDISl et al., 1990)
La presencia de especies como Acanthocyclops robustus, Daphnia ambigua y Daphnia parvula se ha citado como característica de sistemas eutróficos (LACROIX \& LESCHER-MOUTOUE, 1991).

Notodiaptomus incompositus, la especie más abundante, es, con frecuencia, el único Calanoide presente en otros lagos de América del Sur (PAGGI, 1976 ; FALLAVENA, 1985) y, en su éxito, no parece aventurado suponer que factores ligados a una elevada selectividad por el alimento y reducida vulnerabilidad a la depredación por vertebrados (ELMORE et al., 1983) hayan tenido una importancia decisiva. Además, en este lago la población muestra una elevada relación hembras: machos ( $3: 1)$ que, como sugieren OLIVIER (1961) y MONTU (1980), tendería a asegurar un ritmo reproductivo alto que se mantiene durante todo el ciclo anual.

En la predominancia de formas de pequeño y mediano tamaño, es posible evidenciar los efectos derivados de una presión de depredación sobre formas mayores. De hecho, en este sistema se ha descrito (ANONIMO, 1986) la presencia de Oligosarcus hepsetus (dentudo), Hoplias malabaricus (tararira), Cichlaurus facetum (castañeta), Cnesterodon decenmaculatus (overito) y Jenynsia lineata (madrecita) que, en su mayoría, se consideran peces planctófagos facultativos, así como la de elevadas densidades de estadios larvarios de Chaoborus (SOMMARUGA, 1987), un reputado depredador invertebrado (FEDORENKO, 1975; LUECKE \& LITT, 1987; STENSON, 1990).

El posible efecto depredador de A. robustus sobre Rotíferos, nauplios y primeros estadios copepodíticos de Copépodos, que en otros sistemas constituyen la base de su alimento (CRYER \& TOWNSEND, 1989; MAIER, 1990), no ha podido ser evidenciado en el lago Ton-Ton ni siquiera a través de inferencias indirectas basadas en la evolución temporal de la densidad poblacional de esta especie y la de sus presas potenciales.

La depredación, como fuerza estructuradora de la comunidad zooplanctónica, puede reclamarse para explicar las variaciones observadas en el tamaño poblacional de dos especies próximas de similares requerimientos ecológicos y diferente tamaño corporal (Ceriodaphnia dubia y Ceriodaphnia cornuta) (ZARET, 1972) y, por otro lado, es posible interpretar, como suponen LACROIX \& LESCHERMOUTOUE (1984), la diapausa estival de A. robustus, como una estrategia de evitación de la depredación.

La mayoría de los grupos de zooplancton observados en este lago, muestran un típico patrón bimodal de abundancia estacional caracterizado por la presencia de máximos poblacionales en primavera ( $\mathbf{y}$, en menor medida, en otoño) y 
de mínimos en verano (PENNAK, 1946 ; RUTTNER, 1963). Tan sólo los Cladóceros presentan un pulso adicional en Febrero que se debe fundamentalmente a la presencia de Diaphanosoma birgei, una especie descrita como termófila (HUTCHINSON, 1967). En este grupo, además, Cerioduphnia dubia y Bosmina huaronensis muestran pulsos alternados de abundancia a lo largo del período de estudio, interpretables, siguiendo a BURNS \& MITCHELL (1980) y a MATSUMURA-TUNDISI (1985) como resultado de una estrategia de alivio de la competencia interespecífica.

Los pulsos de abundancia de zooplancton registrados en primavera y en otoño, se corresponden con el predominio de especies algales nanoplanctónicas, valores mínimos de concentración de clorofila (DE LEÓN, 1983) y máximos de transparencia. Esto contrasta con la situación estival en la que floraciones de cianobacterias se corresponden con mínimos valores de densidad de zooplancton. Si bién el impacto de pastoreo del zooplancton en la reducción de la biomasa fitoplanctónica parece ser evidente y buen número de trabajos lo corroboran (GLIWICZ, 1977 ; LANE, 1979 ; LAMPERT et al., 1986), no se puede descartar que sobre (junto a) la capacidad de aclaramiento del zooplancton (que, en estos momentos está dominado por especies de pequeño tamaño y de «malla fina») jueguen un papel esencial otros factores como la reducción en el suministro de nutrientes o un incremento en la tasa de «pérdida» algal (sedimentación, parasitismo...) (GELLER \& MÜLLER, 1981 ; REYNOLDS, 1984) de entre los cuales, nuestros resultados sólo permiten visualizar los primeros (Tabla 1).

Autores como MATSUMURA-TUNDISI \& TUNDISI (1976) y ROCHA et al. (1982), han destacado la influencia (y el control) de la precipitación sobre las fluctuaciones estacionales del zooplancton en lagos tropicales y subtropicales. En nuestro caso, esta influencia no se evidencia claramente debido, probablemente, a que en esta región las precipitaciones no son tan marcadamente estacionales como ocurre en el trópico.

Los resultados que hemos obtenido en una (primera) cuantificación del patrón de distribución horizontal del zooplancton, ponen de manifiesto que el efecto del viento como causa de la agregación (MATSUMURA-TUNDISI et al., 1975; PAGGI, 1976) no parece evidente, bien por la moderada/baja intensidad de los vientos predominantes, como por las reducidas dimensiones (fetch) del sistema. La heterogeneidad observada en la distribución vertical, ligada a los períodos de estratificación térmica, ya había sido puesta de manifiesto (FABIAN \& SCHINCA, 1990).

Precisamente, la diferenciación entre períodos de estratificación y mezcla turbulenta en el ciclo estacional de funcionamiento del sistema, parece explicar y dar sentido a la distribución de variables físicas, químicas y biológicas que se observó en el primer eje del análisis de componentes principales llevado a cabo. Los procesos ligados a la actividad de los organismos y, en particular, del fitoplancton pueden explicar la discriminación de variables en el segundo eje que, por lo tanto, estaría relacionado con la productividad del sistema y la distribución estacional de las precipitaciones, parece definir el tercer eje.

\section{BIBLIOGRAFÍA}

ANÓNIMO,1986. Estudio preliminar sobre la población nectónica del lago Ton-Ton. Informe de avance. Dpto. Limnología, Univ. de la República. Uruguay. $11 \mathrm{pp}$.

APHA, 1989. Standard Methods for the Examination of Water and Wastewater. 17 ed. APHA/AWWA/WPCF. Washington. $1484 \mathrm{pp}$.

BLANCHER, C.E. 1984. Zooplankton-trophic state relationships in some north and central Florida lakes. Hydrobiología, 109: 251-263.

BROOKS, J.L. \& S.I. DODSON, 1965. Predation, body size and composition of plankton. Science, 150: 28-35.

BURNS, C.W. \& S.P.MITCHELL, 1980. Seasonal succession and vertical distribution of zooplankton in lake Hayes and lake Johnson. South Island. New Zealand. N. Z. J. Marine Freshwat. Res., 14 (2):189-204.

CANZANI, G. \& R. VARELA, 1984. Introducción al estudio ecológico del zooplancton de la laguna Briozzo (Dpto. Rocha, Uruguay). Tesis de Licenciatura. Univ. de la República. Uruguay. $157 \mathrm{pp}$.

CRYER, M. \& C.R. TOWNSEND, 1989. Generation time of Acanthocyclops robustus in relation to food availability and temperature in a shallow eutrophic lake. Hydrobiología, 182: 93-97.

DE LEÓN, L. 1993. Fitoplancton de un sistema eutrófico. Composición y variación anual de su biomasa. Tesis de Licenciatura. Univ. de la República. Uruguay. 83 pp.

ELMORE, J.L., D.S. VODOPICH \& J.J. HOOVER, 1983. Selective predation by bluegill sunfish (Lepomis macrochirus) on three species of Diaptomus (Copepoda) from subtropical Florida. J.Freshwater Ecol. 2: $183-$ 192.

FABIAN, D. 1993. Composición, distribución horizontal y variación estacional de los Crustáceos planctónicos en el reservorio del Cisne-Uruguay. Rev. Brasil. Biol. 53(3): 355363 
FABIÁN, D \& A. SCHINCA, 1990. Distribución vertical de los Cladóceros en el lago Ton-Ton: diferencias entre los períodos de estratificación y mezcla. Rev. Asoc. Ciens. Nat. Litoral. 21 (2): 1-11.

FALLAVENA, M.A.B. 1985. Composicao e variacoes sazonal e espacial dos Copepodos planctonicos (CrustaceaCopepoda) na lagoa Negra, Municipio de Viamao, Río Grande do Sul, Brasil. Iheringia. 65: 3-39.

FEDORENKO, A.Y. 1975. Instar and species-specific diets in two species of Chaoborus. Limnol. Oceanogr. 20: 238249.

GANNON, J.E \& R. STEMBERGER, 1978. Zooplankton (especially crustaceans and rotífers) as indicator of water quality. Trans. am. microsc. Soc. 97: 16- 35.

GELLER, W. \& H. MÜLLER, 1981. The filtration apparatus of Cladocera: filter mesh sizes and their implication on food selectivity. Oecología (Berlín) 49: 316-321.

GLIWICZ, Z.M. 1977. Food size selection and seasonal succession of filter feeding zooplankton in a eutrophic lake. Ekol. Pol. 25: 179 -225.

HANEY, J.F. \& D.J. HALL. 1973. Sugar-coated Daphnia: a preservation technique for Cladocera. Limnol. Oceanogr. 18: 331-333.

HOLM-HANSEN, O. \& B. RIEMAN, 1978. Chlorophyll a determination: improvement in methodology. Oikos 30 (3): 438-447.

HRBACEK, J., M. DVORAKOVA, V. KORINEK \& L. PROCHAZKOVA, 1961. Demonstration of the effect of the fish stock on the species composition of zooplankton and the intensity of metabolism of the whole plankton association. Verh. Int. Verein. Limnol. 14: 192-195.

HUTCHINSON, G.E. 1967. A treatise on Limnology. Vol II. Introduction to lake biology and the limnoplankton. John Wiley \& Sons. New York. 1115 pp.

LACROIX, G. \& F. LESCHER-MOUTOUE, 1984. Diapause des Cyclopoides d'un écosystème lacustre peu profound (lac de Créteil, France). Annls. Limnol. 20: 183-192.

LACROIX, G \& F. LESCHER-MOUTOUÉ, 1991. Specific composition of microcrustacean planktonic coinmunities in various sand pit lakes: Is competition able to explain the observed pattems?. Verh. Int. Verein. Limnol. 24: 1530-1535.

LAMPERT, W., W. FLECKNER, H. RAI \& B.E. TAYLOR, 1986. Phytoplankton control by grazing zooplankton: A study on the spring clear-water phase. Limnol. Oceanogr. 31: 478-490.

LANE, P.A. 1979. Vertebrate and invertebrate predation intensity of freshwater zooplankton communities. Nature 280: 391-393.
LEWIS, W.M.Jr. 1983. A revised classification of lakes based on mixing. Can. J. Fish. Aquat. Sci. 40: 1779-1787.

LUECKE, C \& A.H. LITT, 1987. Effects of predation by Chaoborus flavicans on crustacean zooplankton of lake Lenore, Washington. Freshwat. Biol. 18: 185-203.

MAIER, G. 1990. Coexistence of the predatory cyclopoids Acanthocyclops robustus (Sars) and Mesocyclops leuckarti (Claus) in a small eutrophic lake. Hydrobiologia, 198: 185203.

MARGALEF, R. 1983. Limnología. Omega. Barcelona. 1010 pp.

MATSUMURA-TUNDISI, T. 1985. Composition and vertical distnbution of zooplankton in lake D. Helvecio (Minas Gerais. Brasil). In: SAIJO, Y. \& J.G. TUNDISI (eds.), Limnological Studies in Central Brasil-Rio Doce Valley Lakes and Pantanal Wetlands. Nagoya University. Nagoya: 129-140.

MATSUMURA-TUNDISI, T. \& J.G. TUNDISI, 1976. Plankton studies in a lacustrine environment. I. Preliininary data on zooplankton ecology of Broa reservoir. Oecologia, 25: 265-270.

MATSUMURA-TUNDISI, T., J.G. TUNDISI \& C.E. MATHEUS, 1975. Plankton studies in a lacustrine environment. II. Spatial distribution of the zooplankton. Cienc. Cult. 27(3): 269-271.

MATSUMURA-TUNDISI, T., A.C. RIETZLER, \& J.G. TUNDISI, 1989. Biomass (dry weight and carbon content) of plankton Crustacea from Broa reservoir (S.Carlos, SPBrazil) and its fluctuation across one year. Hidrobiología, 179: 229-236.

MATSUMURA-TUNDISI, T., S.N. LEITAO, L.S. AGUENA, \& J. MIYAHARA, 1990. Eutrofizaçao da represa de Barra Bonita: Estrutura e organizaçao da comunidade de Rotifera. Rev. Brasil. Biol. 50 (4): 923-935.

MONTÚ, M. 1980. Zooplancton do estuario da Lagoa dos Patos. I. Estructura e variaçoes temporais e espaciais da comunidade. Atlantica. 4: 53-72.

OLIVIER, S.R. 1961. Estudios limnológicos en la laguna Vittel (Pdo. Chascomús). Agro. 3(6): 1-28.

ORCUTT, J.D. \& M.L. PACE, 1984. Seasonal dynamics of rotifer and crustacean zooplankton populations in a eutrophic monomictic lake with a note on rotifer sampling technique. Hydrobiologia, 119: 73-80.

PAGGI, S.J. 1976. Distribución espacial y temporal del zooplancton de un cuerpo de agua eutrófico (Lago del Parque Gral. Belgrano, Santa Fe). Physis (secc. B). 35 (91): 171-183.

PENNAK, R.W. 1946. The dynamics of freshwater plankton populations. Ecol. Monogr. 16: 339-356. 
PENNAK, R.W. 1957. Species composition of limnetic zooplankton communities. Limnol. Oceanogr. 2: 222232.

REYNOLDS, C.S. 1984. The Ecology of Freshwater Phytoplankton. Cambridge. Univ. Press. Cambridgc. 384 pp.

ROCHA, O.,T. MATSUMURA-TUNDISI \& J.G. TUNDISI, 1982. Seasonal fluctuations of Argyrodiaptomus furcatus population in Lobo reservoir (Sao Carlos, SP-Brasil) Tropical Ecology, 23(1): 134-50.

RUTTNER, F. 1963. Fundamentals of Limnology. Toronto Univ. Press. Toronto. 307 pp.

SHANNON, C.E \& W. WEAVER, 1963. The Mathematical Theory of Communication Univ. Illinois Press. Urbana. 177 pp.

SOKAL, R \& F.RHOLF, 1981. Biometry. The Principles and Practice of Statistics in Biological Reseurch. Freeman. San Francisco. 859 pp.

SOMMARUGA, R. 1987. Dinámica de la interfase agua-sedimento en uti sistema eutrófico, lago TonTon (Canelones-Uruguay) con unu caracterización físico-química y biológica del sedimento. Tesis de Licenciatura. Univ.de la República. Uruguay. 93 $\mathrm{pp}$.

SORENSEN, T. 1948. A method of stablishing groups of equal amplitude in a plant sociology based on similarity of species content and its application to analysis of the vegetation on Danish commons. Biol. Skr. 5: 1-34.

STENSON, J.E. 1990. Creating conditions for changes in prey community structure by Chaoborus spp. in a lake in Sweden. Hydrobiologia, 198: 205-214.

WETZEL, R.G. 1983. Limnology. $2^{\text {nd }}$ ed. Saunders Coll., Philadelphia. 860 pp.

WETZEL, R.G. \& G.E. LIKENS, 1991. Limnological Analyses. $2^{\text {nd }}$ ed. Springer-Verlag. New York. 391 pp.

ZARET, T.M. 1972. Predators, invisible prey and the nature of polymorphism in the Cladocera. Limnol. Oceanogr. 17: 171-184. 